\title{
Kinetic Study of the Hydrogen Oxidation Reaction on Membrane Coated Electrodes. Part I: Theoretical Aspects
}

\author{
C.A. Marozzi, M.R. Gennero de Chialvo and A. C. Chialvo* \\ Programa de Electroquímica Aplicada e Ingeniería Electroquímica (PRELINE), Facultad de Ingeniería Química, \\ Universidad Nacional del Litoral, Santiago del Estero 2829, 3000 Santa Fe, Argentina
}

\begin{abstract}
A generalized theoretical analysis of the kinetics of the hydrogen oxidation reaction under the VolmerHeyrovsky-Tafel mechanism on an electrode covered by polymeric films is presented. The treatment includes the diffusion of the molecular hydrogen through the electrolyte solution, as well as through the different layers and interfaces. On the light of the resulting expressions, the applicability of the Levich-Koutecky diagrams for the determination of the kinetic current densities was critically analyzed.
\end{abstract}

Keywords: Hydrogen oxidation, membrane coated electrode, kinetic treatment.

\section{INTRODUCTION}

The fuel cell with solid polymer electrolyte (SPFC) is considered the most appropriate device as an energy source for electric vehicles [1]. However, its implementation remains a costly alternative, which requires new development to make it competitive. This should address both technical (of design) and basic (electrocatalysts, membrane, etc.) aspects [2]. One of the basic problems is related to the kinetics of the electrode reactions, the oxygen reduction reaction (orr) and the hydrogen oxidation reaction (hor), particularly when hydrogen is contaminated with traces of carbon monoxide [3]. For kinetic studies, the electrocatalysts are usually covered by a protonic conductor membrane to reproduce the conditions observed in the fuel cell. In most cases a unique membrane was used [4-10], but recently new arrangements with a multilayer membrane were proposed [11-13]. However, the formalism for an appropriate interpretation of these results has not been developed yet.

The elucidation of these issues requires the understanding of the phenomena that occur at the interface electrocatalystconducting polymer (electronic and mass transfers, etc.). Therefore the study of the aspects that govern the kinetics and the comprehension of the basic processes that occur during the reaction will produce advances in the design of new electrocatalysts, more efficient and cheaper. In this sense, studies carried out in our laboratory [14-19] in aqueous solution demonstrated that the hydrogen electrode reaction on platinum is preferentially verified through the Volmer-Tafel route at low overpotentials and that it is slowly changed to the Volmer-Heyrovsky route as the overpotential is increased. Nevertheless, it should be necessary to carry out basic kinetic studies in conditions similar to those corresponding to the operative cell. Thus, the rigorous

*Address correspondence to this author at the Programa de Electroquímica Aplicada e Ingeniería Electroquímica (PRELINE), Facultad de Ingeniería Química, Universidad Nacional del Litoral, Santiago del Estero 2829, 3000 Santa Fe, Argentina; Tel: +54 342 4571164;

E-mail: achialvo@fiq.unl.edu.ar kinetic analysis of this reaction in electrodes covered with conducting polymeric layers should be of interest taking into account the importance of the diffusional processes in the experimental current-potential response. The solubility $\left(C_{\mathrm{i}}\right)$ and the diffusion coefficient $\left(D_{\mathrm{i}}\right)$ of the hydrogen gas are intrinsic factors of each phase (aqueous electrolytic solution and conducting polymers). For instance the solubility in a Nafion membrane is greater than in aqueous media, while the diffusion coefficient is less in Nafion than in aqueous solutions [4] Furthermore, the product $C_{\mathrm{i}} \times D_{\mathrm{i}}$ as well as the thickness of the diffusion layer $(\delta)$ of both, membrane film and aqueous phase, have an important effect on the experimental current-potential curve. This problem affects the determination of the kinetic parameters on membrane coated electrodes.

Experimental conditions that ensure a steady and quantifiable thickness for the diffusion layer in the electrolyte solution $\left(\delta^{E S}\right)$ are currently used for bare electrodes in order to determine the activated (or faradaic) contribution for reactions with high diffusion effect, from which the kinetic parameters are evaluated. The faradaic contribution is usually obtained from the relationship between current density $(j)$ and $\delta^{E S}$ at constant overpotential $(\eta)$, through a Levich-Koutecky plot [20],

$$
\frac{1}{j\left(\eta, \delta^{E S}\right)}=\frac{1}{j^{k i n}(\eta)}+\frac{1}{j_{L}\left(\delta^{E S}\right)}
$$

where $j_{L}$ is the limiting diffusion current density and $j^{\text {kin }}$ is the faradaic current density. Nevertheless, it has been recently demonstrated that the usual analysis of the hor by this procedure can lead to errors in the interpretation of the kinetic results $[14,17]$. This is mainly due to the variation of the surface coverage $(\theta)$ of the adsorbed intermediate with the thickness of the diffusion layer at constant overpotential.

In the case of an electrode covered with a membrane film there is another diffusion layer, which thickness is equal to that of the membrane itself $\left(\delta^{\mathrm{F}}\right)$. Consequently, for the interpretation of the experimental current-potential curves, the diffusion process inside the membrane must be 
considered. This has been carried out through an extension of the Levich-Koutecky equation, incorporating a term corresponding to the diffusion in the membrane film $[5,8]$,

$\frac{1}{j\left(\eta, \delta^{E S}, \delta^{F}\right)}=\frac{1}{j^{k i n}(\eta)}+\frac{1}{j_{L}\left(\delta^{E S}\right)}+\frac{\delta^{F}}{n F C^{F} D^{F}}$

On this context, the present work deals with a generalized theoretical analysis of the kinetics of the hydrogen oxidation reaction under the Volmer-HeyrovskyTafel mechanism on an electrode covered by a multilayer membrane. In a second part, the resulting kinetic expressions will be applied in order to analyze experimental results obtained on platinum electrodes covered by a Nafion layer.

\section{THEORETICAL TREATMENT}

It is considered a plane electrode with a roughness factor $\left(f_{r}=A_{r} / A_{g}\right)$ and free of edge effects, coated with $n$ polymeric films $\left(F_{i}, i=1,2, \ldots, n\right)$, each with a given constant thickness $\left(\delta^{F_{i}}\right)$, as it can be observed in Fig. (1). The electrode is immersed in an electrolyte solution with a bulk concentration of molecular hydrogen $C_{H_{2}}^{o}$. The number $n+1$ corresponds to the diffusion layer in the electrolyte solution, which thickness $\left(\delta^{F_{n+1}}\right)$ can be varied for instance through the use of a rotating disk electrode. Molecular hydrogen can diffuse in all layers and all of them have a high capacity for proton migration, so that the resistance is considered negligible in the electrolyte solution and along the $n$ layers. The interface $I_{i}$ is located between $F_{i}$ and $F_{(i+1)}$ and $I_{o}$ is the interface where the reaction takes place. At equilibrium, each polymeric layer has a given molecular hydrogen concentration $C_{\mathrm{H}_{2}}^{F_{i}^{e}}(i=$ $1,2,3, \ldots . ., n)$ and that of the electrolyte solution is
$C_{H_{2}}^{F_{n+1}^{e}}=C_{H_{2}}^{o}$.

In the equilibrium conditions, the concentrations at both sides of each interface are related by the corresponding equilibrium constant $K_{i}$,

$K_{i}=\frac{C_{H_{2}}^{F_{i}^{e}}}{C_{H_{2}}^{F_{(i+1)}^{e}}} i=1,2,3, \ldots \ldots, n$

If the potential of the covered electrode is referred to a hydrogen reference electrode (HRE) located in the electrolyte solution, at equilibrium condition both electrodes have the same potential value. As the potential is modified, taking into account the high conductivity of the proton ions through the different layers, its variation against HRE will give directly the corresponding overpotential $(\eta)$ of the hydrogen oxidation reaction.

\subsection{Hydrogen Diffusion}

When the electrode coated with $n$ layers is away from the equilibrium condition and the electric current reaches the value corresponding to the steady state, a consumption of molecular hydrogen and a production of protons take place at the interface between the electrode and the first layer $\left(I_{o}\right)$. Therefore, $\mathrm{H}_{2}$ must be displaced from the bulk of the solution toward the reaction surface, passing through the diffusion layer of the electrolyte solution and the $n$ polymeric layers. Meanwhile $\mathrm{H}^{+}$ions must move in the opposite direction. As a result, $\mathrm{H}_{2}$ concentration gradients are produced within each layer, which will be the driving force for its movement. Assuming that in all phases the $\mathrm{H}_{2}$ diffusion coefficient is constant and independent of the concentration, that the diffusion process occurs only in the direction perpendicular to the electrode and applying Fick's

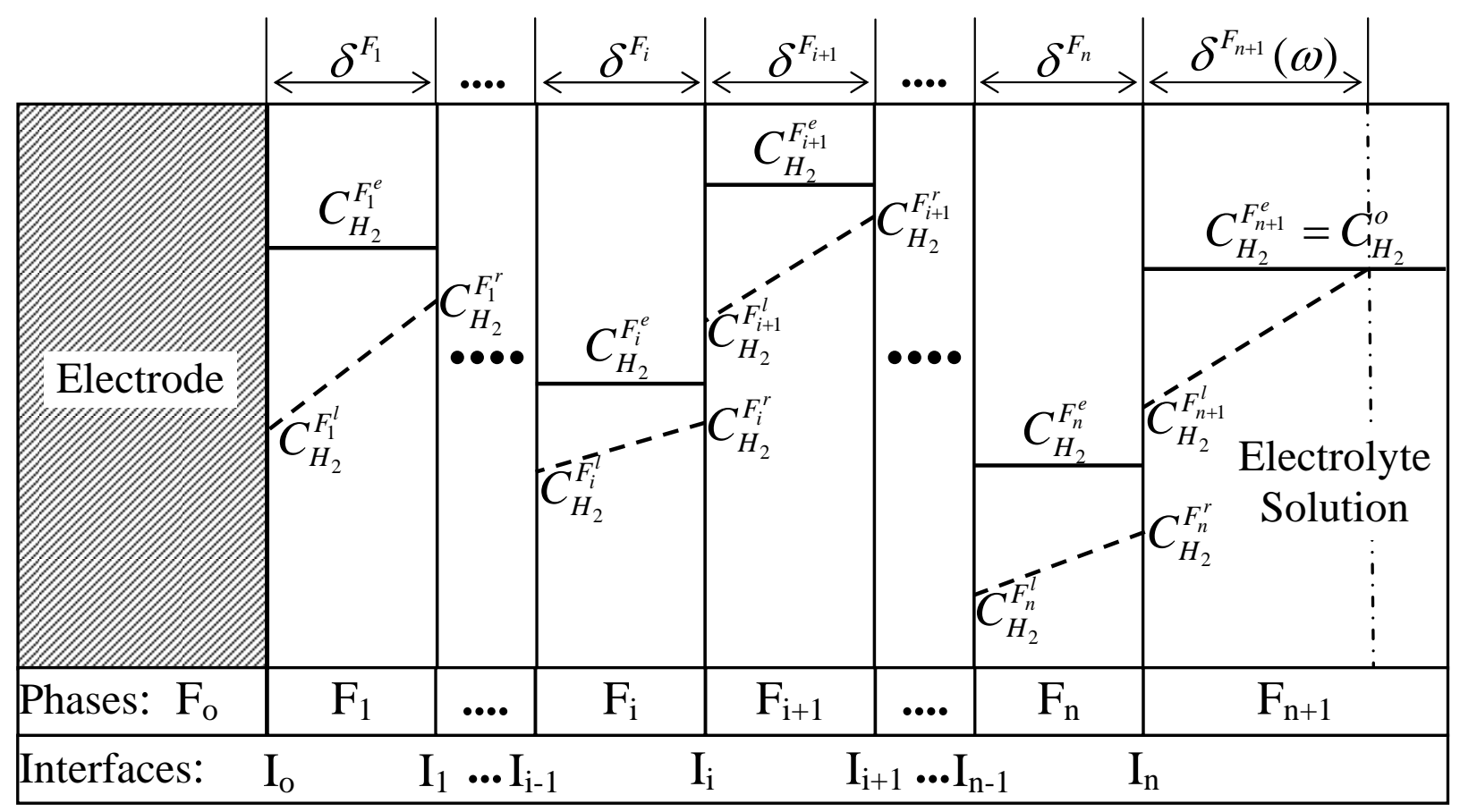

Fig. (1). Scheme of the system under analysis. 
first law, the following expression for the $\mathrm{H}_{2}$ flux in a generic layer $F_{i}\left(J_{H_{2}}^{F_{i}}\right)$ is obtained:

$J_{H_{2}}^{F_{i}}=\frac{D_{H_{2}}^{F_{i}}\left(C_{H_{2}}^{F_{i}^{r}}-C_{H_{2}}^{F_{i}^{l}}\right)}{\delta^{F_{i}}} i=1,2,3, \ldots, n$

where $D_{H_{2}}^{F_{i}}$ is the $\mathrm{H}_{2}$ diffusion coefficient, $C_{H_{2}}^{F_{i}^{l}}$ is the $\mathrm{H}_{2}$ concentration in the left side (interface $I_{i-1}$ ), $C_{H_{2}}^{F_{i}^{r}}$ is the $\mathrm{H}_{2}$ concentration in the right side (interface $I_{i}$ ) and $\delta^{F_{i}}$ is the thickness, all values corresponding to the layer $F_{i}$.

Furthermore, for the process of $\mathrm{H}_{2}$ diffusion in the electrolyte solution it is assumed that the fluid dynamics of the system are such that the model of the Nernstian diffusion layer can be adopted. It assumes that the decrease in concentration is produced in a layer of thickness $\delta^{F_{n+1}}$, uniform and constant over time, beyond which the concentration remains constant and equal to that of the bulk solution. In these conditions, the $\mathrm{H}_{2}$ flux in the electrolyte solution $\left(J_{H_{2}}^{F_{n+1}}\right)$ is given by:

$J_{H_{2}}^{F_{n+1}}=\frac{D_{H_{2}}^{F_{n+1}}\left(C_{H_{2}}^{o}-C_{H_{2}}^{F_{n+1}^{l}}\right)}{\delta^{F_{n+1}}}$

where $D_{H_{2}}^{F_{n+1}}$ is the $\mathrm{H}_{2}$ diffusion coefficient and $C_{H_{2}}^{F_{n+1}^{l}}$ is the $\mathrm{H}_{2}$ concentration in the left side (interface $I_{n}$ ) of the electrolyte solution.

It has been considered that the $\mathrm{H}_{2}$ flux through the different interfaces, from the bulk solution to the electrode surface, is governed by a first order kinetics [21],

$J_{H_{2}}^{I_{i}}=k_{H_{2}}^{F_{(i+1)}^{l}} C_{H_{2}}^{F_{(i+1)}^{l}}-k_{H_{2}}^{F_{i}^{r}} C_{H_{2}}^{F_{i}^{r}} i=1,2,3, \ldots \ldots, n$

where $J_{H_{2}}^{I_{i}}$ is the $\mathrm{H}_{2}$ flux through the interface $I_{i}, k_{H_{2}}^{F_{(i+1)}^{l}}$ is the kinetic constant of the transfer process from the layer $F_{i+1}$ towards $F_{i}, k_{H_{2}}^{F_{i}^{r}}$ is the kinetic constant of the reverse process. At equilibrium conditions $\left(J_{H_{2}}^{I_{i}}=0\right)$ the following expression is fulfilled,

$k_{H_{2}}^{F_{(i+1)}^{l}} \quad C_{H_{2}}^{F_{(i+1)}^{e}}=k_{H_{2}}^{F_{i}^{r}} \quad C_{H_{2}}^{F_{i}^{e}} \quad i=1,2,3, \ldots \ldots, n$

The relationship between the equilibrium constant corresponding to the interface $\mathrm{I}_{\mathrm{i}}$ and the transfer kinetic constants can be obtained taking into account Equation 3,

$K_{i}=\frac{k_{H_{2}}^{F_{(i+1)}^{l}}}{k_{H_{2}}^{F_{i}^{r}}} \quad i=1,2,3, \ldots, n$

At steady-state conditions the $\mathrm{H}_{2}$ flux $\left(J_{H_{2}}\right.$ ) must be the same in all phases and therefore,
$J_{H_{2}}=J_{H_{2}}^{F_{i}}=J_{H_{2}}^{I_{i}}=J_{H_{2}}^{F_{n+1}} \quad i=1,2,3, \ldots, n$

The $\mathrm{H}_{2}$ flux can be related to the current density of the electrochemical reaction $(j)$. It is important to notice that $j$ is referred to the real electrode area $\left(A_{r}\right)$ while $J_{H_{2}}$ is referred to the geometric area $\left(A_{g}\right)$. Therefore, the relationship between them must include the roughness factor of the reaction surface $\left(f_{r}=A_{r} / A_{g}\right)$,

$j f_{r}=2 F J_{H_{2}}$

Then, the relationship between the surface concentration in the reaction plane at a given $j$ value $\left(C_{H_{2}}^{F_{1}^{l}}\right)$, and that corresponding to equilibrium $\left(C_{H_{2}}^{F_{1}^{e}}\right.$ ) can be obtained with the help of Equations (3-10) (see Appendix),

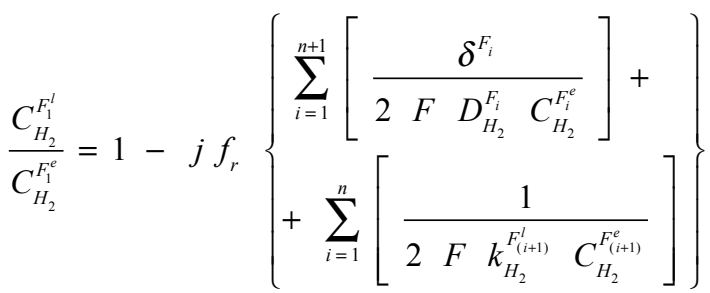

The $\mathrm{H}_{2}$ flux defined by $D_{H_{2}}^{F_{i}} C_{H_{2}}^{F_{i}^{e}} / \delta^{F_{i}}$ is the maximum value for the layer $F_{i}$, as the highest value for $C_{H_{2}}^{F_{i}^{r}}$ is $C_{H_{2}}^{F_{i}^{e}}$ and the lowest value of $C_{H_{2}}^{F_{i}^{l}}$ is zero (Equation 4). Consequently, the limiting diffusion current density of the layer i $\left(j_{L}^{F_{i}}\right)$ can be defined as,

$j_{L}^{F_{i}}=\frac{2 F D_{H_{2}}^{F_{i}} C_{H_{2}}^{F_{i}^{e}}}{\delta^{F_{i}}} i=1,2,3, \ldots \ldots \ldots,(n+1)$

From Equation (6) it can be derived that the product $k_{H_{2}}^{F_{(i+1)}^{l}} C_{H_{2}}^{F_{(i+1)}^{e}}$ is the maximum $\mathrm{H}_{2}$ diffusion flux that can pass through the interface $I_{i}$, as the highest value for $C_{H_{2}}^{F_{(i+1)}^{l}}$ is $C_{H_{2}}^{F_{(i+1)}^{e}}$ and the minimum value of $C_{H_{2}}^{F_{i}^{r}}$ is zero. Consequently, the limiting transfer current density through the interface $i$ $\left(j_{L}^{I_{i}}\right)$ can be defined as,

$j_{L}^{I_{i}}=2 F k_{H_{2}}^{F_{(i+1)}^{l}} C_{H_{2}}^{F_{(i+1)}^{e}} i=1,2,3, \ldots \ldots, n$

Then, Equation (11) can be written as,

$\frac{C_{H_{2}}^{F_{1}^{l}}}{C_{H_{2}}^{F_{1}^{e}}}=1-j f_{r}\left\{\sum_{i=1}^{n+1}\left[\frac{1}{j_{L}^{F_{i}}}\right]+\sum_{i=1}^{n}\left[\frac{1}{j_{L}^{I_{i}}}\right]\right\}$

On the other hand, when $C_{H_{2}}^{F_{1}^{l}}=0$ the highest current density that can circulate for the whole system is achieved. This value is called limiting diffusion current density $\left(j_{L}\right)$ and 
is referred to the geometric area. Applying this condition to Equation (14) and reordering, the expression for the reciprocal of $j_{L}$ is obtained,

$\frac{1}{j_{L}}=\sum_{i=1}^{n+1}\left[\frac{1}{j_{L}^{F_{i}}}\right]+\sum_{i=1}^{n}\left[\frac{1}{j_{L}^{I_{i}}}\right]$

Finally, substituting Equation (15) into Equation (14) the expression for the relationship $C_{H_{2}}^{F_{1}^{l}} / C_{H_{2}}^{F_{1}^{e}}$ can be written as,

$\frac{C_{H_{2}}^{F_{1}^{l}}}{C_{H_{2}}^{F_{1}^{e}}}=1-\frac{j f_{r}}{j_{L}}$

This expression is formally equal to that corresponding to a bare electrode under the model of the Nernst diffusion layer. The difference is that now $j_{L}$ is given by Equation (15), which takes into account the $\mathrm{H}_{2}$ diffusion in the $n$ polymeric films and through the corresponding interfaces.

\subsection{Hydrogen Oxidation Reaction}

The hydrogen oxidation reaction (hor) in acid electrolyte solution is,

$H_{2} \rightleftarrows 2 H^{+}+2 e^{-}$

It takes place in the interface $I_{o}$ through the following kinetic steps,

$$
\begin{aligned}
& H_{2\left(F_{1}\right)}+2 S \rightleftarrows 2 H_{(a)} \quad \text { Tafel } \\
& H_{2\left(F_{1}\right)}+S \rightleftarrows H_{(a)}+H_{\left(F_{1}\right)}^{+}+e^{-} \text {Heyrovsky } \\
& H_{(a)} \rightleftarrows H_{\left(F_{1}\right)}^{+}+e^{-}+S \quad \text { Volmer }
\end{aligned}
$$

where $\mathrm{S}$ is an adsorption site and $\mathrm{H}_{(\mathrm{a})}$ is the adsorbed hydrogen. The expressions for both the current density $(j)$ and the surface coverage of the adsorbed hydrogen $(\theta)$ as a function of overpotential $(\eta)$ for the simultaneous occurrence of the three steps under a Frumkin type adsorption were derived previously [17]. For the present case the equations for $j(\eta)$ can be written as,

$$
\begin{gathered}
j=2 F\left\{v_{T}^{e}\left[\begin{array}{ll}
\left(\frac{1-\theta}{1-\theta^{e}}\right)^{2} & e^{-2 u\left(\theta-\theta^{e}\right) \lambda}\left(\frac{C_{H_{2}}^{F_{1}^{l}}}{C_{H_{2}}^{F^{e}}}\right)- \\
-\left(\frac{\theta}{\theta^{e}}\right)^{2} e^{2 u\left(\theta-\theta^{e}\right)(1-\lambda)}
\end{array}\right]\right. \\
\left.+v_{H}^{e}\left[\begin{array}{ll}
\left(\frac{1-\theta}{1-\theta^{e}}\right) e^{-u\left(\theta-\theta^{e}\right) \lambda} & \left.\frac{C_{H_{2}}^{F_{1}^{l}}}{C_{H_{2}}^{F^{e}}}\right) e^{\alpha_{H} f \eta}- \\
-\left(\frac{\theta}{\theta^{e}}\right) e^{u\left(\theta-\theta^{e}\right)(1-\lambda)} & e^{-\left(1-\alpha_{H}\right) f \eta}
\end{array}\right]\right\}
\end{gathered}
$$

$$
\begin{aligned}
& j=2 F\left\{v_{V}^{e}\left[\begin{array}{l}
\left(\frac{\theta}{\theta^{e}}\right) e^{u\left(\theta-\theta^{e}\right)(1-\lambda)} e^{\alpha_{V} f \eta}- \\
-\left(\frac{1-\theta}{1-\theta^{e}}\right) e^{-u\left(\theta-\theta^{e}\right) \lambda} e^{-\left(1-\alpha_{V}\right) f \eta}
\end{array}\right]\right.
\end{aligned}
$$

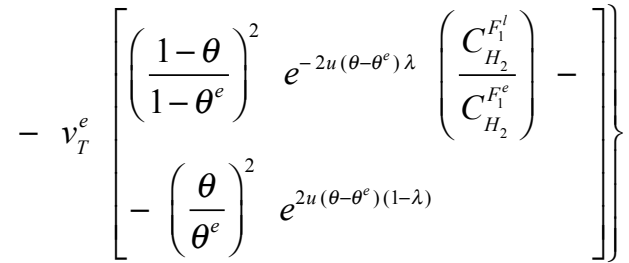

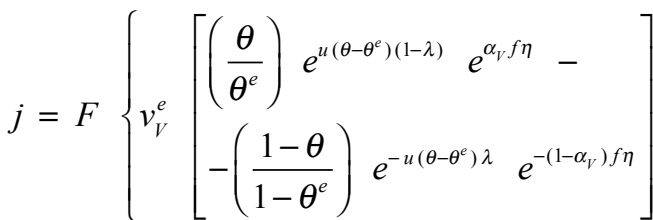

$$
\begin{aligned}
& \left.+v_{H}^{e}\left[\begin{array}{ll}
\left(\frac{1-\theta}{1-\theta^{e}}\right) e^{-u\left(\theta-\theta^{e}\right) \lambda} & \left(\frac{C_{H_{2}}^{F^{l}}}{C_{H_{2}}^{F^{e}}}\right) e^{\alpha_{H} f \eta}- \\
-\left(\frac{\theta}{\theta^{e}}\right) e^{u\left(\theta-\theta^{e}\right)(1-\lambda)} & e^{-\left(1-\alpha_{H}\right) f \eta}
\end{array}\right]\right\}
\end{aligned}
$$

where $v_{i}$ is the rate of the elementary step $i(i=V, H, T), \alpha_{i}$ is the symmetry factor of the elementary step $i(i=V, H), u$ (in RT units) and $\lambda$ are Frumkin adsorption parameters, $f=$ $F / R T$ and the superscript $e$ indicates equilibrium conditions. It can be observed in these expressions that the influence of the $\mathrm{H}_{2}$ diffusion on $j$ is given by the quotient $C_{H_{2}}^{F_{1}^{l}} / C_{H_{2}}^{F_{1}^{e}}$, which is described by Equation (16). Substituting it into Equation (19) and reordering, the final expressions for the dependences $j(\eta)$ are obtained:

$$
\begin{aligned}
& j=\frac{v_{T}^{e}\left[\begin{array}{c}
\left(\frac{1-\theta}{1-\theta^{e}}\right)^{2} e^{-2 u\left(\theta-\theta^{e}\right) \lambda}- \\
-\left(\frac{\theta}{\theta^{e}}\right)^{2} e^{2 u\left(\theta-\theta^{e}\right)(1-\lambda)}
\end{array}\right]}{\left[\begin{array}{l}
\left.\frac{1}{2 F}+\frac{v_{T}^{e}}{\left(j_{L} / f_{r}\right)}\left(\frac{1-\theta}{1-\theta^{e}}\right)^{2} e^{-2 u\left(\theta-\theta^{e}\right) \lambda}+\right] \\
+\frac{v_{H}^{e}}{\left(j_{L} / f_{r}\right)}\left(\frac{1-\theta}{1-\theta^{e}}\right) e^{-u\left(\theta-\theta^{e}\right) \lambda} e^{\alpha_{H} f \eta}
\end{array}\right]} \\
& +\frac{v_{H}^{e}\left[\begin{array}{l}
\left(\frac{1-\theta}{1-\theta^{e}}\right) e^{-u\left(\theta-\theta^{e}\right) \lambda} e^{\alpha_{H} f \eta}- \\
\left.-\left(\frac{\theta}{\theta^{e}}\right) e^{u\left(\theta-\theta^{e}\right)(1-\lambda)} e^{-\left(1-\alpha_{H}\right) f \eta}\right]
\end{array}\right.}{\left[\frac{1}{2 F}+\frac{v_{T}^{e}}{\left(j_{L} / f_{r}\right)}\left(\frac{1-\theta}{1-\theta^{e}}\right)^{2} e^{-2 u\left(\theta-\theta^{e}\right) \lambda}+\right.} \\
& +\frac{v_{H}^{e}}{\left(j_{L} / f_{r}\right)}\left(\frac{1-\theta}{1-\theta^{e}}\right) e^{-u\left(\theta-\theta^{e}\right) \lambda} e^{\alpha_{H} f \eta}
\end{aligned}
$$




$$
\begin{gathered}
j=\frac{v_{V}^{e}\left[\left(\frac{\theta}{\theta^{e}}\right) e^{u\left(\theta-\theta^{e}\right)(1-\lambda)} e^{\alpha_{V} f \eta}-\left(\frac{1-\theta}{1-\theta^{e}}\right) e^{-u\left(\theta-\theta^{e}\right) \lambda} e^{-\left(1-\alpha_{V}\right) f \eta}\right]}{\left[\frac{1}{2 F}-\frac{v_{T}^{e}}{\left(j_{L} / f_{r}\right)}\left(\frac{1-\theta}{1-\theta^{e}}\right)^{2} e^{\left.-2 u\left(\theta-\theta^{e}\right) \lambda\right]}\right]} \\
-\frac{v_{T}^{e}\left[\left(\frac{1-\theta}{1-\theta^{e}}\right)^{2} e^{-2 u\left(\theta-\theta^{e}\right) \lambda}-\left(\frac{\theta}{\theta^{e}}\right)^{2} e^{2 u\left(\theta-\theta^{e}\right)(1-\lambda)}\right]}{\left[\frac{1}{2}-\frac{v_{T}^{e}}{\left(j_{L} / f_{r}\right)}\left(\frac{1-\theta}{1-\theta^{e}}\right)^{2} e^{-2 u\left(\theta-\theta^{e}\right) \lambda}\right]} \\
j=\frac{v_{V}^{e}\left[\left(\frac{\theta}{\theta^{e}}\right) e^{u\left(\theta-\theta^{e}\right)(1-\lambda)} e^{\alpha_{V} f \eta}-\left(\frac{1-\theta}{1-\theta^{e}}\right)^{-u\left(\theta-\theta^{e}\right) \lambda} e^{-\left(1-\alpha_{V}\right) f \eta}\right]}{\left[\frac{1}{F}+\frac{v_{H}^{e}}{\left(j_{L} / f_{r}\right)}\left(\frac{1-\theta}{1-\theta}\right) e^{-u\left(\theta-\theta^{e}\right) \lambda} e^{\alpha_{H} f \eta}\right]} \\
+\frac{v_{H}^{e}\left[\left(\frac{1-\theta}{1-\theta^{e}}\right) e^{-u\left(\theta-\theta^{e}\right) \lambda} e^{\alpha_{H} f \eta}-\left(\frac{\theta}{\theta^{e}}\right) e^{u\left(\theta-\theta^{e}\right)(1-\lambda)} e^{-\left(1-\alpha_{H}\right) f \eta}\right]}{\left[\frac{v_{H}^{e}}{\left(j_{L} / f_{r}\right)}\left(\frac{1-\theta}{1-\theta}\right) e^{-u\left(\theta-\theta^{e}\right) \lambda} e^{\alpha_{H} f \eta}\right]}
\end{gathered}
$$

The corresponding expression for the dependence $\theta(\eta)$ can be obtained equating two of the Equations $(20 a-c)$ and reordering,

$2 v_{T}^{e}\left[\left(\frac{1-\theta}{1-\theta^{e}}\right)^{2} e^{-2 u\left(\theta-\theta^{e}\right) \lambda}-\left(\frac{\theta}{\theta^{e}}\right)^{2} e^{2 u\left(\theta-\theta^{e}\right)(1-\lambda)}\right]$

$+v_{H}^{e}\left[\left(\frac{1-\theta}{1-\theta^{e}}\right) e^{-u\left(\theta-\theta^{e}\right) \lambda} e^{\alpha_{H} f \eta}-\left(\frac{\theta}{\theta^{e}}\right) e^{u\left(\theta-\theta^{e}\right)(1-\lambda)} e^{-\left(1-\alpha_{H}\right) f \eta}\right]$

$+v_{V}^{e}\left[\left(\frac{1-\theta}{1-\theta^{e}}\right) e^{-u\left(\theta-\theta^{e}\right) \lambda} e^{-\left(1-\alpha_{V}\right) f \eta}-\left(\frac{\theta}{\theta^{e}}\right) e^{u\left(\theta-\theta^{e}\right)(1-\lambda)} e^{\alpha_{V} f \eta}\right]$

$+\left(\frac{1-\theta}{1-\theta^{e}}\right) e^{-u\left(\theta-\theta^{e}\right) \lambda} \frac{2 F}{\left(j_{L} / f_{r}\right)}\left\{\left[\left(\frac{1-\theta}{1-\theta^{e}}\right) e^{-u\left(\theta-\theta^{e}\right) \lambda} v_{T}^{e} v_{V}^{e}+v_{V}^{e} v_{H}^{e} e^{\alpha_{H} f \eta}\right]\right.$

$\left[\left(\frac{1-\theta}{1-\theta^{e}}\right) e^{-u\left(\theta-\theta^{e}\right) \lambda} e^{-\left(1-\alpha_{Y}\right) f \eta}-\left(\frac{\theta}{\theta^{e}}\right) e^{u\left(\theta-\theta^{e}\right)(1-\lambda)} e^{\alpha_{V} f \eta}\right]$

$+\left[\left(\frac{\theta}{\theta^{e}}\right) e^{u\left(\theta-\theta^{e}\right)(1-\lambda)} v_{T}^{e} v_{H}^{e}\right]$

$\left.\left[\left(\frac{1-\theta}{1-\theta^{e}}\right) e^{-u\left(\theta-\theta^{e}\right) \lambda} e^{-\left(1-\alpha_{H}\right) f \eta}-\left(\frac{\theta}{\theta^{e}}\right) e^{u\left(\theta-\theta^{e}\right)(1-\lambda)} e^{\alpha_{H} f \eta}\right]\right\}=0$

The dependence $j(\eta)$ can be simulated by the simultaneous resolution of one of the Equations (20a-c) and (21). The corresponding kinetic parameters are $j_{L}, v_{i}^{e}(i=V$, $H, T), \theta^{e}, u, \lambda$ and $\alpha$, for a given electrode of surface roughness $f_{r}$. It should be noticed that $j_{L}$ depends on the $\mathrm{H}_{2}$ diffusion through all the layers and interfaces. The analysis of the effect of the hydrogen solubility in each layer will be discussed in the second part of the present work.

\section{DISCUSSION}

The present work deals with the derivation of the analytical expressions for the description of the currentpotential dependence of the hydrogen oxidation reaction on electrodes coated with ion-conducting membranes and immersed in an electrolyte solution operating on steady state. The resulting expressions reveal that there are important differences with respect to those corresponding to bare electrodes.

\subsection{Analysis of Equation (15)}

Equation (15) shows that the reciprocal of the limiting diffusion current density is the result of two types of contributions. The first term is the summation over the reciprocals of the limiting diffusion current densities of each layer, while the second term corresponds to the summation over the reciprocals of the limiting transfer current density through each interface. It should be noted that this expression is not specific for the hor, as it can be applied to any electrode reaction in similar conditions, for instance to the oxygen reduction reaction on electrodes covered with a Nafion membrane.

Equation (15) can be compared with those used in previous kinetic studies, for the case in which there is only one polymeric film $[17,19]$. The difference is that the limiting current density in the interface between the film and the electrolyte solution was not taken into account. Therefore for those works, Equation (2) can be more appropriately written as,

$$
\frac{1}{j\left(\eta, \delta^{F_{1}}, \delta^{F_{2}}\right)}=\frac{1}{j^{k i n}(\eta)}+\frac{1}{j_{L}^{F_{1}}\left(\delta^{F_{1}}\right)}+\frac{1}{j_{L}^{I_{1}}}+\frac{1}{j_{L}^{F_{2}}\left(\delta^{F_{2}}\right)}
$$

being $F_{1}$ the polymeric film and $F_{2}$ the diffusion layer in the electrolyte solution. Equation (22) can be only reduced to the usual form if the limiting current density of the interface is infinite and therefore it does not offer any resistance to the transfer of matter.

\subsection{Analysis of Equation (20)}

The kinetic description of the hydrogen oxidation reaction at the interface electronic conductor-polymer membrane $\left(I_{o}\right)$ was given considering that the adsorbed intermediate $\mathrm{H}_{(\mathrm{a})}$ has a Frumkin type behaviour, as it was verified previously [17]. The dependences $\theta\left(\eta, j_{L}\right)$ and $j(\eta$, $j_{L}$ ) are formally equal to those corresponding to the case of an uncovered electrode, being the difference in the expression of $j_{L}$, which takes into account not only the hydrogen diffusion in the electrolyte solution but also in the $\mathrm{n}$ layers and through the corresponding interfaces. Consequently the kinetic behaviour is formally identical to the uncoated electrode for the same values of the parameters involved, including $j_{L}$ [17]. In this context, for the case in which the hor takes place preferably through the VolmerTafel route, a maximum value of the current density $j_{\text {max }}$ will be obtained when $\eta \rightarrow \infty$, which can be less or equal to $j_{L}$ depending on the values of parameters $j_{L}, v_{T}^{e}$ and $\theta^{e}$ (Equations $7 \mathrm{a}-8$ in reference 16). Moreover, for the Heyrovsky-Volmer route $j_{\max }$ will be equal to $j_{L}$ when $\eta \rightarrow$ $\infty$, regardless of the values of all the parameters [14-16]. In the case of the simultaneous occurrence of the three steps, depending on the relative values of the different parameters, $j(\eta)$ can exhibit a first zone at low $\eta$ determined by the Tafel step and then a gradual increase in the contribution of the Heyrovsky step, which finally becomes preponderant [14, 16]. 


\subsection{Levich-Koutecky Plots}

The Levich-Koutecky diagrams consist in the plot of $1 / j\left(\eta, j_{L}\right)$ vs. $1 / j_{L}$, at a given $\eta$ value. It is presupposed linear so that at the intercept the reciprocal of the kinetic current density $j^{k i n}(\eta)^{-1}$ could be obtained, free of any diffusion contribution. However in the case of the hor, the reordering of any of the Equations (20a-c) leads to,

$$
\frac{1}{j\left(\eta, j_{L}\right)}=a\left(\eta, j_{L}\right)+b\left(\eta, j_{L}\right) \frac{1}{\left(j_{L} / f_{r}\right)}
$$

where,

$$
\begin{aligned}
& a\left(\eta, j_{L}\right)=\left\{\begin{array}{lll}
2 F & v_{T}^{e}
\end{array}\left[\left(\frac{1-\theta}{1-\theta^{e}}\right)^{2} e^{-2 u\left(\theta-\theta^{e}\right) \lambda}-\left(\frac{\theta}{\theta^{e}}\right)^{2} e^{2 u\left(\theta-\theta^{e}\right)(1-\lambda)}\right]\right. \\
& \left.+2 F v_{H}^{e}\left[\left(\frac{1-\theta}{1-\theta^{e}}\right) e^{-u\left(\theta-\theta^{e}\right) \lambda} e^{\alpha_{H} f \eta}-\left(\frac{\theta}{\theta^{e}}\right) e^{u\left(\theta-\theta^{e}\right)(1-\lambda)} e^{-\left(1-\alpha_{H}\right) f \eta}\right]\right\}^{-1} \\
& b\left(\eta, j_{L}\right)=\left[v_{T}^{e}\left(\frac{1-\theta}{1-\theta^{e}}\right)^{2} e^{-2 u\left(\theta-\theta^{e}\right) \lambda}+v_{H}^{e}\left(\frac{1-\theta}{1-\theta^{e}}\right) e^{-u\left(\theta-\theta^{e}\right) \lambda} e^{\alpha_{H} f \eta}\right] \\
& \left\{v_{T}^{e}\left[\left(\frac{1-\theta}{1-\theta^{e}}\right)^{2} e^{-2 u\left(\theta-\theta^{e}\right) \lambda}-\left(\frac{\theta}{\theta^{e}}\right)^{2} e^{2 u\left(\theta-\theta^{e}\right)(1-\lambda)}\right]\right. \\
& \left.+v_{H}^{e}\left[\left(\frac{1-\theta}{1-\theta^{e}}\right) e^{-u\left(\theta-\theta^{e}\right) \lambda} e^{\alpha_{H} f \eta}-\left(\frac{\theta}{\theta^{e}}\right) e^{u\left(\theta-\theta^{e}\right)(1-\lambda)} e^{-\left(1-\alpha_{H}\right) f \eta}\right]\right\}^{-1}
\end{aligned}
$$

Equation (23) is similar to that previously deduced for an uncovered electrode, but now $j_{L}$ is given by Equation (15) and it depends on the properties of all the layers which are present in the system. It can be appreciated that the dependence $1 / j\left(\eta_{j} j_{L}\right)$ vs. $1 / j_{L}$ can only be linear in the case in which $\theta\left(\eta, j_{L}\right)$ is invariant on $j_{L}$, depending only on $\eta$. It can be considered that when the current density reaches the value $j_{\max }$, the surface coverage is $\theta \cong 0$ and thus independent of $j_{L}$. Therefore, the plot $1 / j_{\max }$ vs. $1 / j_{L}$ is strictly linear and Equation (23) can be written as,

$\frac{1}{j_{\max }\left(j_{\mathrm{L}}\right)}=a_{\max }+b_{\max } \frac{1}{\left(j_{\mathrm{L}} / f_{r}\right)}$

The Levich-Koutecky diagrams are usually drawn from experimental data obtained through the use of a rotating disk electrode. In this case the limiting diffusion current density of the electrolyte solution is proportional to $\omega^{1 / 2}$, being $\omega$ the rotation rate. If the rotating disk is uncovered, at constant overpotential, for $\omega^{1 / 2} \rightarrow \infty$ it is satisfied that $j_{L}=j_{L}^{F_{n+1}} \rightarrow \infty$ and thus $1 / j_{L} \rightarrow 0$. Then, from the extrapolation of the plot $1 / j_{\max }$ vs. $\omega^{-1 / 2}$, for $\omega^{-1 / 2} \rightarrow 0$, the true value of the maximum kinetic current density $j_{\max }^{k i n}$ is obtained $\left(a_{\max }{ }^{-1}=j_{\max }^{k i n}\right)$. On the other hand, if the rotating disk is covered with $n$ layers, the condition $\omega^{-1 / 2} \rightarrow 0$ leads to $1 / j_{L}^{F_{n+1}} \rightarrow 0$ and therefore $1 / j_{L}$

$\rightarrow 1 / j_{L}^{*}$, where $j_{L}^{*}$ is,

$$
\frac{1}{j_{L}^{*}}=\sum_{i=1}^{n}\left[\frac{1}{j_{L}^{F_{i}}}\right]+\sum_{i=1}^{n}\left[\frac{1}{j_{L}^{I_{i}}}\right]=\frac{1}{j_{L}(\omega \rightarrow \infty)}
$$

Consequently, the intercept in the Levich-Koutecky diagram does not correspond in this case to the reciprocal of $j_{\max }^{k i n}$, but a value $\left(j_{\max }^{*}{ }^{-1}\right)$ that includes the limiting diffusion current density of the $\mathrm{n}$ polymeric layers and the limiting transfer current density through the $\mathrm{n}$ interfaces.

The diffusion contribution of the $\mathrm{n}$ layers could in principle be eliminated through the application of limit conditions to the layers thicknesses $\left(\Sigma \delta^{\mathrm{Fi}} \rightarrow 0\right)$, but the diffusion contribution of the $\mathrm{n}$ interfaces cannot be eliminated. Therefore, yet in the simplest case in which $\theta \cong 0$ and $j(\eta)=\mathrm{j}_{\max }$, the true value of $j_{\max }^{k i n}$ cannot be obtained by the method of Levich-Koutecky. For $\eta$ values where $\theta=\theta\left(\eta, j_{L}\right)$, it will be even more complicated to try to determine the true values of the kinetic current density $j^{\text {kin }}(\eta)$. Therefore, the use of these diagrams for covered electrodes is not advisable.

\section{CONCLUSIONS}

A kinetic analysis of the hydrogen electrode reaction on electrodes coated with $\mathrm{n}$ layers of polymer films and immersed in an electrolyte solution containing $\mathrm{H}_{2}$ and $\mathrm{H}^{+}$ ions was carried out. The expressions of the dependences $\theta\left(\eta, j_{L}\right)$ and $j\left(\eta, j_{L}\right)$ are formally equal to those corresponding to a bare electrode. The main difference is in the expression of the limiting diffusion current density $j_{L}$, which now includes not only the diffusion contribution of the electrolyte solution, but also those corresponding to the $n$ layers and interfaces. On the other hand, the applicability of the LevichKoutecky diagrams for the determination of the kinetic current densities was also analyzed and discussed. It was concluded that such method cannot be applied to the present case. Consequently it is considered that for the determination of the kinetic parameters of the hor it should be more appropriate the correlation of the experimental curves $j\left(\eta, j_{L}\right)$ with the kinetic equations, as it will be illustrated in Part II of the present work.

Finally, the fundamental concepts involved in the proposed formalism can be used for the analysis of other kind of experimental results carried out on electrodes covered by a multilayer membrane.

\section{ACKNOWLEDGEMENTS}

The authors gratefully acknowledge financial support given by ANPCyT, CONICET and UNL.

\section{APPENDIX}

In order to derive Equation (11), the following expression is obtained applying the condition $n=1$ to Equation (4) an reordering,

$C_{H_{2}}^{F_{1}^{l}}=C_{H_{2}}^{F_{1}^{r}}-\frac{J_{H_{2}}^{F_{1}} \delta^{F_{1}}}{D_{H_{2}}^{F_{1}}}$

An expression for $C_{H_{2}}^{F_{2}^{r}}$ can be obtained in turn from Equation (6) and taking also into account Equation (8),

$$
C_{H_{2}}^{F_{1}^{r}}=\frac{k_{H_{2}}^{F_{2}^{l}}}{k_{H_{2}}^{F_{1}^{r}}} C_{H_{2}}^{F_{2}^{l}}-\frac{J_{H_{2}}^{I_{1}}}{k_{H_{2}}^{F_{1}^{r}}}=K_{1} C_{H_{2}}^{F_{2}^{l}}-K_{1} \frac{J_{H_{2}}^{I_{1}}}{k_{H_{2}}^{F_{2}^{l}}}
$$


Substituting $C_{H_{2}}^{F_{2}^{l}}$ by the expression obtained applying the condition $\mathrm{n}=2$ to Equation (4) and taking also into account Equation (10),

$C_{H_{2}}^{F_{1}^{r}}=K_{1}\left\{C_{H_{2}}^{F_{2}^{r}}-\frac{j f_{r}}{2 F}\left[\frac{\delta^{F_{2}}}{D_{H_{2}}^{F_{2}}}+\frac{1}{k_{H_{2}}^{F_{2}^{l}}}\right]\right\}$

A similar expression for $C_{H_{2}}^{F_{i}^{r}}(i=2,3, \ldots, n)$ can be obtained through the same procedure,

$C_{H_{2}}^{F_{i}^{r}}=K_{i}\left\{C_{H_{2}}^{F_{i+1}^{r}}-\frac{j f_{r}}{2 F}\left[\frac{\delta^{F_{i+1}}}{D_{H_{2}}^{F_{i+1}}}+\frac{1}{k_{H_{2}}^{F_{i+1}^{l}}}\right]\right\}$

Substituting successively $C_{H_{2}}^{F_{2}^{r}}, C_{H_{2}}^{F_{3}^{r}}, \ldots, \quad C_{H_{2}}^{F_{n}^{r}}$ into Equation (A3) and then the resulting expression into Equation (A1),

$C_{H_{2}}^{F_{1}^{\prime}}=\left(\prod_{j=1}^{n} K_{j}\right) C_{H_{2}^{\prime}}^{F_{n+1}^{r}}-\frac{j f_{r}}{2 F}\left\{\sum_{i=1}^{n+1}\left(\prod_{j=0}^{i-1} K_{j}\right) \frac{\delta^{F_{i}}}{D_{H_{2}}^{F_{j}}}+\sum_{i=1}^{n}\left(\prod_{j=1}^{i} K_{j}\right) \frac{1}{k_{H_{2}}^{F_{t+1}^{t}}}\right\}$

Taking into account Equation (3), it is obtained that,

$$
\begin{aligned}
& \prod_{i=1}^{n} K_{i}=K_{1} K_{2} \ldots \ldots . K_{n}=\frac{C_{H_{2}}^{F_{1}^{e}}}{C_{H_{2}}^{F_{2}^{e}}} \frac{C_{H_{2}}^{F_{2}^{e}}}{C_{H_{2}}^{F_{3}^{e}}} \ldots \ldots \ldots \frac{C_{H_{2}}^{F_{n}^{e}}}{C_{H_{2}}^{F_{(n+1)}^{e}}}=\frac{C_{H_{2}}^{F_{1}^{e}}}{C_{H_{2}}^{F_{(n+1)}^{e}}}=\frac{C_{H_{2}}^{F_{1}^{e}}}{C_{H_{2}}^{o}} \\
& \prod_{j=1}^{i} K_{j}=K_{1} K_{2} \ldots . K_{i}=\frac{C_{H_{2}}^{F_{1}^{e}}}{C_{H_{2}}^{F_{2}^{e}}} \frac{C_{H_{2}}^{F_{2}^{e}}}{C_{H_{2}}^{F_{3}^{e}}} \ldots \ldots . . \frac{C_{H_{2}}^{F_{i}^{e}}}{C_{H_{2}}^{F_{(j+1)}^{e}}}=\frac{C_{H_{2}}^{F_{1}^{e}}}{C_{H_{2}}^{F_{(i+1)}^{e}}} \\
& \prod_{j=0}^{i-1} K_{j}=K_{0} K_{1} \ldots K_{i-1}=1 \frac{C_{H_{2}}^{F_{1}^{e}}}{C_{H_{2}}^{F_{2}^{e}}} \frac{C_{H_{2}}^{F_{2}^{e}}}{C_{H_{2}}^{F_{3}^{e}}} \ldots \ldots . . . \frac{C_{H_{2}}^{F_{i-1}^{e}}}{C_{H_{2}}^{F_{i}^{e}}}=\frac{C_{H_{2}}^{F_{1}^{e}}}{C_{H_{2}}^{F_{i}^{e}}}
\end{aligned}
$$

where it was considered $K_{0}=1$. Equation (11) is obtained substituting Equations (A6), (A7) and (A8) into Equation (A5), dividing it by $C_{H_{2}}^{F_{1}^{e}}$ and taking into account that $C_{H_{2}}^{o}=C_{H_{2}}^{F_{n+1}^{r}}$.

\section{REFERENCES}

[1] Pei, P.; Chang, Q.; Tang, T. A quick evaluating method for automotive fuel cell lifetime. Int. J. Hydrogen Energy, 2008, 33, 3829-36.

[2] Srinivasan, S. Fuel Cells: From Fundamentals to Applications. Springer: New York 2006.

[3] Springer, T. E.; Rockward, T.; Zawodzinski, T. A.; Gottesfeld, S. Model for Polymer electrolyte fuel cell operation on reformate feed: effects of $\mathrm{CO}, \mathrm{H} 2$ dilution, and high fuel utilization. $J$. Electrochem. Soc., 2001, 148, A11-23.

[4] Ogumi, Z.; Kuroe, T.; Takehara, Z. Gas permeation in SPE method. II. Oxygen and hydrogen permeation through nafion. $J$. Electrochem. Soc., 1985, 132, 2601-5.

[5] Lawson, D. R.; Whiteley, N.; Martin, C. R. Oxygen reduction at Nafion film-coated platinum electrodes: Transport and kinetics. $J$. Electrochem. Soc., 1988, 135, 2247-53.

[6] Watanabe, M.; Igarashi, H.; Yosioka, K. An experimental prediction of the preparation condition of Nafion-coated catalyst layers for PEFCs. Electrochim. Acta, 1995, 40, 329-34.

[7] Lin, R. B.; Shih, S. M. Kinetic analysis of the hydrogen oxidation reaction on Pt-black/Nafion electrode. J. Sol. State Electrochem., 2006, 10, 243-9.

[8] Maruyama, J.; Inaba, M.; Katakura, K.; Ogumi, Z.; Takehara, Z. Influence of Nafion ${ }^{\circledR}$ film on the kinetics of anodic hydrogen oxidation. J. Electroanal. Chem., 1998, 447, 201-9.

[9] Mello, R. M. Q.; Ticianelli, E. A. Kinetic study of the hydrogen oxidation reaction on platinum and Nafion covered platinum electrodes. Electrochim. Acta, 1997, 42, 1031-9.

[10] Higuchi, E.; Uchida, H.; Watanabe, M. Effect of loading level in platinum-dispersed carbon black electrocatalysts on oxygen reduction activity evaluated by rotating disk electrode. $J$. Electroanal. Chem., 2005, 583, 69-76.

[11] Deligöz, H.; Yılmaztürk, S.; Karaca, T.; Özdemir, H.; Koc, S. N.; Öksüzömer, F.; Durmus, A.; Gürkaynak, M. A. Self-assembled polyelectrolyte multilayered films on Nafion with lowered methanol cross-over for DMFC applications. J. Membrane Sci., 2009, 326, 643-9.

[12] Yang, T.; Zhang, S. X.; Gao, Y.; Ji, F. C.; Liu, T. W. Multilayer membranes based on sulfonated poly(ether ether ketone) and poly(vinyl alcohol) for direct methanol membrane fuel cells. Open Fuel Cells J., 2008, 1, 4-8.

[13] Jiang, S. P.; Liu, Z.; Tian, Z. Q. Layer-by-layer self-assembly of composite polyelectrolyte-nafion membranes for direct methanol fuel cells. Adv. Mater., 2006, 18, 1068-72.

[14] Gennero-Chialvo, M. R.; Chialvo, A. C. Hydrogen diffusion effects on the kinetics of the hydrogen electrode reaction. Part I. Theoretical aspects. Phys. Chem. Chem. Phys., 2004, 6, 4009-17.

[15] Quaino, P. M.; Gennero-Chialvo, M. R.; Chialvo, A. C. Hydrogen diffusion effects on the kinetics of the hydrogen electrode reaction. Part II. Evaluation of kinetic parameters. Phys. Chem. Chem. Phys., 2004, 6, 4450-5.

[16] Quaino, P. M.; Fernandez, J. L.; Gennero-Chialvo, M. R.; Chialvo, A. C. Hydrogen oxidation reaction on microelectrodes: Analysis of the contribution of the kinetic routes. J. Mol. Catalisis A: Chem., 2006, 252, 156-62.

[17] Quaino, P. M.; Gennero-Chialvo, M. R.; Chialvo, A. C. Hydrogen electrode reaction: A complete kinetic description. Electrochim. Acta, 2007, 52, 7396-403.

[18] Rau, M. S.; Quaino, P. M.; Gennero-Chialvo, M. R.; Chialvo, A. C. Hydrogen oxidation reaction: Evidences of different electrocatalytic activity between alpha and beta Pd-H. Electrochem. Commun., 2008, 10, 208-12.

[19] Quaino, P. M.; Gennero-Chialvo, M. R.; Chialvo, A. C. Kinetic study of the hydrogen electrode reaction on Bi-modified platinum. J. Electrochem. Soc., 2009, 156, B167-73.

[20] Jahn, D.; Vielstich, W., Rate of electrode processes by the rotating disk method. J. Electrochem. Soc., 1962, 109, 849-52.

[21] Leddy, J.; Bard, A. J.; Maloy, J. T.; Saveant, J. M. Kinetics of filmcoated electrodes. Effect of a finite mass transfer rate of substrate across the film-solution interface at steady state. J. Electroanal. Chem., 1985, 187, 205-27. 